\title{
Processos de Projeto Cibersemióticos: Procedimentos de Observação, Representação e Performance Aplicados ao Design Paramétrico
}

\author{
Cybersemiotic Design Processes: Observation, Representation and Performance Proceedings Applied on \\ Parametric Design
}

\author{
Gilfranco Alves \\ Universidade de São Paulo, Brazil \\ gilfranco@sc.usp.br
}

\author{
Anja Pratschke \\ Universidade de São Paulo, Brazil \\ pratschke@sc.usp.br
}

\begin{abstract}
The abstract presented here is part of a PhD research in progress, currently developed at USP - University of São Paulo, with the Nomads.usp research group, under the guidance of Professor Anja Pratschke. This research starts from the assumption that Cybersemiotics, initially defined by the Philosopher and Information Scientist Dr. Søren Brier - and therefore gathers concepts of Second Order Cybernetics and Semiotics of Charles Sanders Peirce - while meta-theory designed and developed to promote transdisciplinarity, can bring other perspectives and base updated reflections in order to produce a framework for architectural design processes that make use of digital mediation, especially Parametric Design.
\end{abstract}

Keywords: Cybersemiotics; Cybernetics; Peircean semiotics; Performance; Parametric design.

\section{Introdução}

As recentes possibilidades de mediação digital utilizadas nos projetos arquitetônicos têm sido observadas e analisadas por vários estudiosos da Arquitetura Contemporânea, o que os tem levado a considerar mudanças no método de criação arquitetônica, na medida em que se percebem outras maneiras possíveis no ato de se projetar. É possível afirmar que há uma tendência em nível mundial na arquitetura contemporânea, que aponta para a consolidação de um modo de se encarar o desafio projetivo que está modificando os processos de projeto concebidos em ambiente digital: o Design Paramétrico ou Parametricismo.

Poderíamos descrever o Design Paramétrico como sendo um método fundamentado em técnicas de criação e modelagem digital, a partir de algorítimos e sistemas de programação que antecipam a tomada de decisões para uma etapa bastante inicial do processo de projeto - a fase de definição dos parâmetros (inputs). Um parâmetro pode ser entendido como o valor de uma variável que, ao mudar, fornece uma característica diferente ao componente, mas mantém uma relação tipológica indicial com este componente em seu estado original.

\section{Design Paramétrico para Quem Precisa!}

De acordo com Neil Leach ${ }^{1}$ (s.d.), o Design Paramétrico encontrase atualmente a conquistar sua hegemonia, talvez sucedendo o Modernismo como o próximo momento histórico relevante de inovação sistemática significativa para a Arquitetura, em termos

\footnotetext{
${ }^{1}$ Neil Leach é um arquiteto e teórico. Atualmente leciona na Universidade da Califórnia do Sul e lecionou na University of Brighton, University of Bath, Architectural Association School of Architecture, entre outros. Fonte: wikipedia: http://en.wikipedia.org/wiki/Neil_Leach. Acessado em: 15/08/2013.
}

de método (LEACH, s/d p.34). Ainda segundo o autor, o Design Paramétrico promove uma ruptura e finaliza uma fase de transição e incerteza gerada pela crise do Modernismo e que foi marcada por uma série de episódios arquitetônicos de busca por outros caminhos com duração relativamente curta. Estes caminhos incluíam, por exemplo, o Pós-modernismo, o Desconstrutivismo e o Minimalismo, estilos arquitetônicos decorrentes do meio produtivo da própria Arquitetura Moderna.

Não compartilhamos integralmente dessa visão otimista e pragmática, assim como não imaginamos como um processo evolutivo dinâmico poderia ser retratado de modo estanque. A transição para processos de projetos híbridos envolve saberes e procedimentos baseados nas relações entre indivíduos e algorítimos e estas são relações ainda muito recentes, se comparadas à história da própria arquitetura.

Pedro Fiori Arantes alerta para os riscos da fetichização da tecnologia e da mera utilização das possibilidades de mediação digital para buscas estritamentes formais. Segundo ele, "A pesquisa formal é autorreferente, dobra-se sobre si mesma de modo autista, complexificando a geometria e simplificando as relações sociais e urbanas do entorno, anulando o tempo histórico, apagando contadições e conflitos." (ARANTES, 2012 p.171).

Porém, acreditamos que o processo de projeto possibilitado pelo Design Paramétrico favorece a colaboração criativa e pode ser alimentado por inputs socias, parâmetros inseridos no sistema, de baixo para cima (bottom-up). Michael Meredith escreve sobre a questão: "Em última análise, o que nos interessa sobre o projeto paramétrico é exatamente o que ele exclui, a dimensão sóciopolítica da arquitetura. O potencial do Paramétrico é produzir uma 
rede hyperinclusiva de parâmetros e relacionamentos - quanto mais polivalente o objeto, mais significativo e complexo ele é." (MEREDITH, 2008 p.08 Tradução nossa. ${ }^{2}$ )

Apresentamos aqui brevemente, uma experimentação que envolveu o uso do Design Paramétrico e que trabalhou com a alimentação do sistema a partir de inputs ${ }^{3}$ de ordem sóciocultural. Trata-se da ação Fabricação Digital do projeto Territórios Híbridos: meios digitais, comunidades e ações culturais (processo FAPESP 09/51457-5), desenvolvido pelo grupo de pesquisa Nomads.usp junto à comunidade do CDHU em São Carlos/SP Brasil - em 2012. A partir da criação de um meta-design, equipamentos (computadores equipados com os software Rhino $e$ Grasshopper e telões com fotografias da paisagem local) foram instalados na comunidade e a partir da manipulação de alguns parâmetros, previamente discutidos com os moradores do bairro, cada morador poderia explorar a possibilidade de criação de um pavilhão (posteriormente denominado Slice), inicialmente previsto para abrigar o centro comunitário do referido conjunto habitacional. (figura 1).

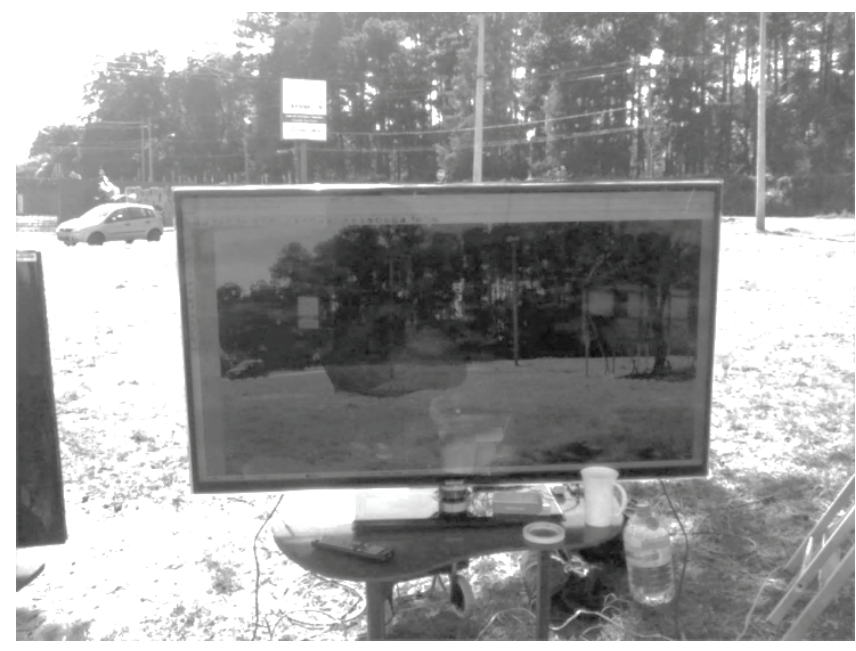

Figura 1: Metadesign na paisagem local - CDHU - São Carlos/SP, Brasil Nomads.usp. Fonte: Autor, 2012.

Este olhar, que evoca uma observação semioticizada para além dos significados meramente relacionados às questões formais, pode ser uma estratégia de atuação dentro do processo de criação. O Design Paramétrico está em evidência em muitas áreas da produção arquitetônica contemporânea, do design de interiores ao desenho urbano de grande escala, e percebe-se em função dessa gama de possibilidades atualmente verificada na prática projetual, que uma de suas maiores qualidades é a sua grande capacidade para articular as mais variadas complexidades.

A Figura 2 mostra a interface computacional (Rhino + Grasshopper) da consequente evolução do projeto do pavilhão

\footnotetext{
${ }^{2}$ Do original em inglês: "Ultimately, what interest us about the parametric project is exactly what it excludes, the socio-political dimension of architecture. Parametric's potencial is to produce a hyperinclusive network of parameters and relationships - the more multivalent the object the more meaningful and complex it is."

${ }^{3}$ Inputs são entradas (de dados) de projeto ou parâmetros.
}

Slice, explorando a espacialidade criada a partir dos parâmetros pré-definidos no design colaborativo realizado na ação.

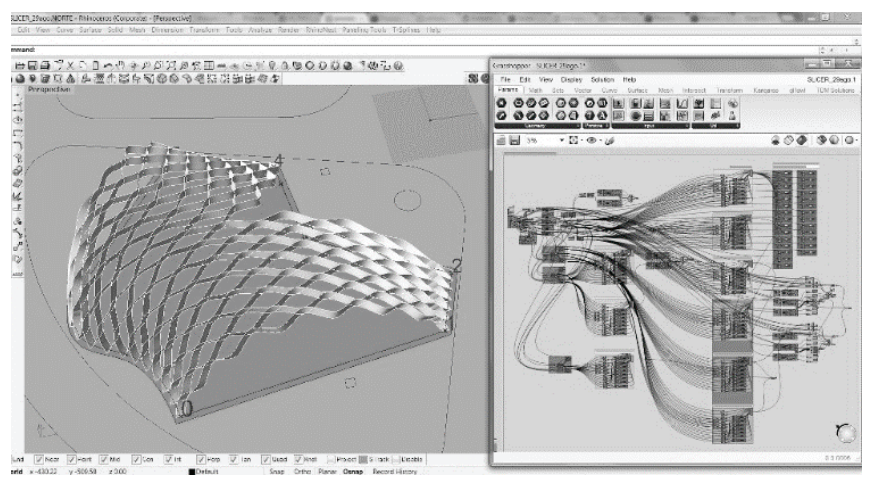

Figura 2: Script: pavilhão Slice - Nomads.usp. Fonte: Autor, 2012.

Outra questão relevante é o fato de que o Design Paramétrico está atualmente recuperando e revendo fundamentos da Arquitetura que estiveram presentes em muitas fases da história da humanidade e que sempre buscaram base em outros campos do conhecimento como, por exemplo, a matemática, a biologia e os estudos da linguagem, esta última compreendida em toda a sua ampla capacidade de comunicação. Sob certo aspecto, os tratados mais antigos da arquitetura, os saberes compartilhados pelos grandes cânones da história, como os textos de Alberti $^{4}$, que traziam em sua essência uma descrição de procedimentos que em muito se assemelhavam a detalhadas instruções acerca de métodos construtivos e proporções a serem adotados, são comparáveis aos scripts ${ }^{5}$ usados no Design Paramétrico, conforme atesta Mario Carpo:

"... textos alfabéticos e números,eram conhecidos por viajar através do espaço e do tempo mais rápido e com mais segurança do que imagens. E, na época de Alberti, a maioria dos estudiosos teriam sabido que a fidelidade de uma cópia à mão de um desenho manuscrito também era inversamente proporcional à complexidade do desenho, e à sua distância a partir do arquétipo. Diagramas geométricos simples, por exemplo, poderiam sobreviver a várias rodadas de cópia relativamente incólumes, mas desenhos mais ricos ou menos geometricamente definíveis não podiam. Textos em ordem alfabética (e sequências numéricas, tanto no Hindú-Árabe como nas notações Latinas) têm uma vantagem sobre os desenhos feitos à mão: letras e números, todos juntos, fazem uma espécie de lista de sinais padronizados exatamente repetitivos: um desenho é feito de um número

${ }^{4}$ Leon Battista Alberti (Génova, 18 de Fevereiro de 1404 - Roma, 20 de Abril de1472) foi um arquiteto, teórico de arte e humanista italiano. Ao estilo do ideal renascentista, foi filósofo da arquitetura e do urbanismo, pintor, músico e escultor.

${ }^{5}$ Um script é um conjunto de instruções para uma determinada ação que o programa ou aplicativo realizará. Fonte: http://www.dicionarioinformal.com.br/script/. 
imprevisível de sinais, nenhum deles padronizado ou exatamente repetitivo ". (CARPO 2011, p. 53. Tradução Nossa ${ }^{6}$ ).

Através da utilização de um sistema de coordenadas polares para traduzir um conteúdo gráfico em listas de coordenadas, Alberti usava o processo de digitalização da informação. A messagens submetidas eram incluídas nas listas de coordenadas em vez da imagem, e também as instruções para traduzir essas coordenadas em um desenho.

Portanto, pode-se dizer que a herança de um saber geométrico advindo do período clássico, é atualmente potencializada por algoritmos e funções matemáticas com altíssima capacidade de cálculo e de construção tridimensional em ambientes digitais, ao mesmo tempo em que as interfaces dos software de Design Paramétrico se tornam cada vez mais amigáveis aos usuários. Deste modo, nos projetos arquitetônicos contemporâneos, uma significativa mudança de ênfase pode ser detectada - um reposicionamento em direção a um aprofundamento maior na questão da performance ${ }^{7}$. Aspectos econômicos, ambientais e sociais, entre outros parâmetros, estão sendo tratados como possíveis inputs de suma importância dentro do processo de projeto desde sua concepção inicial.

\section{Observação, Representação e Performance}

Partindo do contexto acima apresentado, a hipótese que o trabalho explora é de que a Cibernética de Segunda Ordem e a Semiótica de Charles Sanders Peirce ${ }^{8}$, enquanto teorias concebidas e desenvolvidas para promover a transdisciplinaridade, podem trazer outros olhares e fundamentar reflexões atualizadas, no sentido de produzir um framework Cibersemiótico para processos de projeto arquitetônico que se utilizam da mediação digital, especialmente para o Design Paramétrico.

\footnotetext{
${ }^{6}$ Do original em inglês: “... alphabetical texts and numbers, were known to travel across space and time faster and more safely than pictures. And, at the time of Alberti, most scholars would have known that the fidelity of a handmade copy of a manuscript drawing was also in inverse proportion to the complexity of the drawing, and to its distance from the archetype. Simple geometrical diagrams, for example, could survive several rounds of copying relatively unscathed, but richer or less geometrically definable drawings could not. Alphabetical texts (and numerical sequences, both in the Hindu-Arabic and in the Latin notations) have one advantage over handmade drawings: letter and numbers, all together, make for a sort of list of exactly repeatable standardized signs: a drawing is made up of an unpredictable number of signs, none of them standart or exactly repeatable". (CARPO 2011, p. 53).

'Performance: $n 1$ execução, efetuação. 2 cumprimento, desempenho. 3 façanha, proeza. 4 representação, artística, espetáculo. 5 atuação (de artista, atleta, etc.). 6 Tech capacidade de trabalho mecânico, rendimento. Fonte: Dicionário Michaelis.

${ }^{8}$ Charles Sanders Peirce (Cambridge, Massachussets, 1839-1914) era um cientista. Bacharelado em Química aos 11 anos de idade, era também matemático, físico, astrônomo, além de ter realizado contribuições importantes nos campos da Geodésia, Metrologia e Espectroscopia. Era ainda um estudioso da Biologia e da Geologia. No campo das ciências culturais, se devotou à Linguística, à Filosofia e à História. Fonte: Wikipedia: http://pt.wikipedia.org/wiki/Charles_Sanders_Peirce, acessado em 01/10/2013.
}

Para a demonstração dos conceitos e a evolução da pesquisa, concebeu-se um diagrama (Figura 3) que visa proporcionar uma visualização esquemática dos conhecimentos envolvidos, além de representar as devidas aproximações e imbricações entre os diferentes campos de conhecimento.

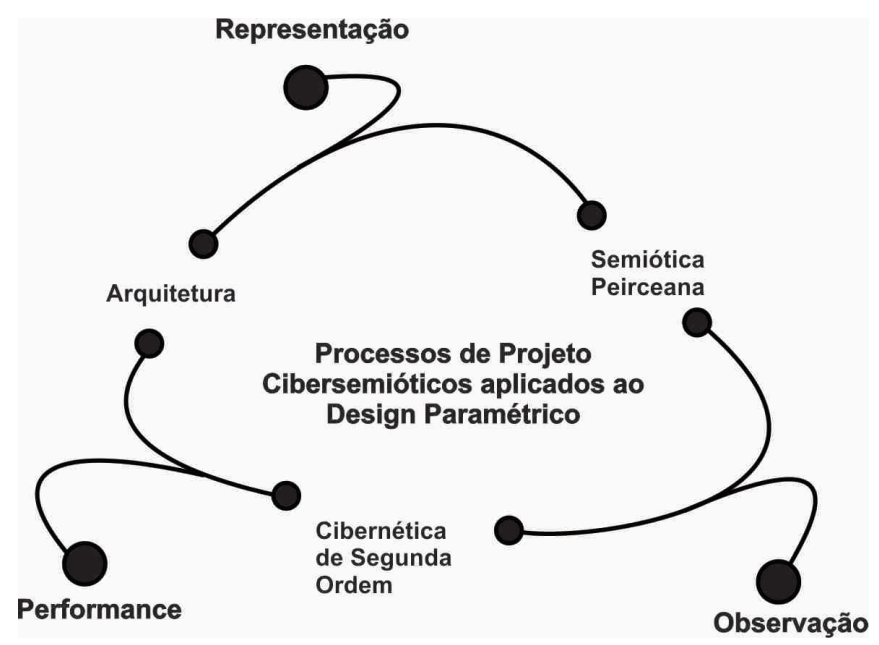

Figura 3: Diagrama - Processos de Projeto Cibersemióticos aplicados ao Design Paramétrico Fonte: autor, 2013.

O diagrama representa um modo de explicar esquematicamente as relações estabelecidas, delimitando os campos de conhecimento abordados e indicando desdobramentos em relação às aproximações que são propostas entre eles, aos pares, em função de pontos em comum identificados e recortados. Propõese então, que a partir das sobreposições dos três campos de conhecimento envolvidos como elementos principais: Arquitetura, Semiótica Perciana e Cibernética de Segunda Ordem, três outros campos de qualidades puras - a partir da noção de primeiridade em Peirce - sejam admitidos como locus de desenvolvimento teórico, em função das características em comum e das possibilidades geradas, ou seja, a observação, a representação e a performance.

Enquanto a observação é um conceito muito importante usado tanto pela Semiótica (em função da fenomenologia de Peirce e da observação dos fenômenos) como pela Cibernética (onde o observador - especialmente o de segunda ordem assume um papel central nas discussões propostas por Heinz von Foerster ${ }^{9}$ ), a representação traz consigo a relação estabelecida entre as questões inerentes aos signos e significados, e o modo como a arquitetura desenvolveu suas linguagens (não verbais) a partir de

9 Heinz von Foerster (13 de novembro de 1911, Viena - 2 de outubro de 2002, Pescadero, California) foi um cientista austríacoamericano que combinava física com filosofia. Juntamente com Warren McCulloch, Norbert Wiener, John von Neumann, Lawrence J. Fogel, Gregory Bateson, Humberto Maturana, Francisco Varela e outros, Heinz von Foerster foi um dos arquitetos da cibernética. Fonte: wikipedia: http://pt.wikipedia.org/wiki/Heinz_von_Foerster. Acessado em 29/09/2013. 
valores expressos nos projetos e nas obras. São estes valores que acreditamos serem fundamentais para a inserção dos mais variados inputs de projeto, inclusive os de ordem social.

A performance, em relação a suas possibilidades de procedimentos em termos de processos Cibersemióticos, traz também para a discussão, a ligação entre Arquitetura e Cibernética, retomando historicamente as teorias dos anos 1960 e que somente agora, com o desenvolvimento de software e de computadores em rede e conectados via internet, além de equipamentos com maior capacidade de desempenho, como por exemplo os de prototipagem rápida e de fabricação digital ${ }^{10}$ permitem que as experimentações propostas nos anos 1960 quase como uma ficção, sejam hoje viabilizadas do ponto de vista da materialização destas experiências.

Conforme afirma Michael Hensel, "Performance em arquitetura tornou-se novamente um discurso vital que pode fornecer uma plataforma relevante para o desenvolvimento de uma abordagem alternativa para a concepção arquitetônica e para a sustentabilidade." (HENSEL, 2012 p.140 Tradução Nossa ${ }^{11}$ ).

\section{Considerações Finais}

A Cibernética de Segunda Ordem aliada à Semiótica de Peirce resulta em uma teoria transdiciplinar - a Cibersemiótica - que se constitui em um desenvolvimento sofisticado de conexão entre muitos conceitos, e que pode apontar caminhos relacionados à percepção (a observação), à intencionalidade significativa (a representação), e à organização e controle de desempenho dos sistemas, possíveis de serem estudados e mimetizados a partir dos sistemas biológicos e do comportamento das máquinas (a performance), proporcionando, portanto, outros olhares e estratégias em relação aos processos de projeto contemporâneos que se utilizam do Design Paramétrico.

É possivel sugerir que para um processo de Design Paramétrico que vise não apenas limitar-se às questões da gênese da forma, seja importante que se definam inicialmente as relações e a vontade do designer de considerar a observação, a representação e a performance como referências estabelecidas de modo a se tornarem partes integrantes de um processo de projeto mais amplo e colaborativo. Isto inicialmente requer que o arquiteto dê um passo para trás em relação à ação propriamente dita do design, esteja aberto à observação dos fenômenos e atento aos seus significados, mude sua atitude em relação à pré-configuração dos parâmetros de entradas do projeto e se concentre na lógica voltada ao desempenho que interliga o projeto, e que envolve além dos participantes, vários campos de conhecimento em suas respectivas complexidades.

\section{Agradecimentos}

Ao Comitê Internacional SIGRADI por fornecer a base para este modelo.

Á FAPESP - Fundação de Amparo à Pesquisa do Estado de São Paulo. (http://www.fapesp.br/)

Ao grupo de pesquisa Nomads.usp. (www.nomads.usp.br)

\section{Referências}

Arantes, Pedro Fiori. Arquitetura na Era Digital-Financeira: Desenho, Canteiro e Renda da Forma. São Paulo: Ed. 34, 2012.

Carpo, Mario. The Alphabet and the Algorithm. Cambridge: MIT, 2011.

Hensel, Michael. Design Innovation for the Built Environment: Research by Design and the Renovation of Practice. New York: Routledge, 2012.

Leach, Neil. Metabolism and Morphology. In Architectural Design s/n. P 3237. Wiley \& Sons, s/d.

Meredith, Michael. From Control to Design. New York: Actar-D, 2008.

Pupo, Regiane Trevisan. A inserção da Prototipagem e Fabricação Digitais no Processo de Projeto: um novo desafio para o ensino de Arquitetura. Campinas, 2008. Tese (Doutorado) - Universidade Estadual de Campinas.

\footnotetext{
${ }^{10}$ Segundo Pupo (2008), prototipagem rápida inclui todas as técnicas de prototipagem de rápida execução (sobreposição de camadas), corte a laser, fresas e corte com vinil, para a produção de maquetes em escalas reduzidas e protótipos em escala 1:1, enquanto a fabricação digital inclui técnicas destinadas à produção de edifícios ou partes deles (file-to-factory, metal e tube bending), estas, por sua vez, destinadas à produção de fôrmas ou peças finais de edifícios, com equipamentos de CNC.

${ }^{11}$ Performance in architecture has once again become a vital discourse that can provide a relevant platform for developing na alternative approach to architectural design and sustainability" (HENSEL 2012, P.140).
} 